\title{
What does inflation say about dark energy given the swampland conjectures?
}

\author{
Chien-I Chiang, ${ }^{1,2, *}$ Jacob M. Leedom, ${ }^{1,2, \dagger}$ and Hitoshi Murayama ${ }^{1,3,2, \$}$ \\ ${ }^{1}$ Department of Physics, University of California, Berkeley, California 94720, USA \\ ${ }^{2}$ Ernest Orlando Lawrence Berkeley National Laboratory, Berkeley, California 94720, USA \\ ${ }^{3}$ Kavli Institute for the Physics and Mathematics of the Universe (WPI), University of Tokyo, \\ Kashiwa 277-8583, Japan
}

(Received 3 December 2018; published 2 August 2019)

\begin{abstract}
We discuss the relations between swampland conjectures and observational constraints on both inflation and dark energy. Using the requirement $|\nabla V| \geq c V$, with $c$ as a universal constant whose value can be derived from inflation, there may be no observable distinction between constant and nonconstant models of dark energy. However, the latest modification of the above conjecture, which utilizes the second derivative of the potential, opens up the opportunity for observations to determine if the dark energy equation of state deviates from that of a cosmological constant. We also comment on the observability of tensor fluctuations despite the conjecture that field excursions are smaller than the Planck scale.
\end{abstract}

DOI: 10.1103/PhysRevD.100.043505

\section{INTRODUCTION}

The discovery of the accelerating expansion of the Universe [1,2] was a huge surprise to the community. Because gravity only pulls, it should hinder the expansion of the Universe after the big bang, and hence the expansion should decelerate. Acceleration implies that there is a substance in the Universe that pushes the expansion. It was dubbed dark energy. The most discussed candidate for dark energy is the cosmological constant $\Lambda$, a finite energy density of the vacuum, due to the simple way it can be implemented into cosmological models based on general relativity. However, despite being consistent with data [3], the 120 orders of magnitude difference between the observed vacuum energy density $\left(\rho \approx(\mathrm{meV})^{4}\right)$ and the naive theoretical expectation $\left(\rho \approx M_{P 1}^{4}\right)$ still remains the most challenging problem in modern physics [4].

Since dark energy and the cosmological constant problem inevitably involve quantum gravity, string theory, as a theory of quantum gravity, should address these topics. The attempts to construct de Sitter solutions (spacetime solutions to general relativity with a positive $\Lambda$ ) in string theory [5-7] have led to the notion of the string landscape.

\footnotetext{
*chienichiang@berkeley.edu

†leedoj@berkeley.edu

"hitoshi@berkeley.edu; hitoshi.murayama@ipmu.jp
}

Published by the American Physical Society under the terms of the Creative Commons Attribution 4.0 International license. Further distribution of this work must maintain attribution to the author(s) and the published article's title, journal citation, and DOI. Funded by SCOAP .
The landscape consists of an enormous number of vacua, each described by different low-energy effective field theories (EFTs) of different fields and parameters. String theory therefore supports the anthropic argument [8], namely, that the value of the observed dark energy density is what it is because otherwise human civilization could not exist. If we really live in a (meta)stable vacuum in the string landscape where a constant vacuum energy explains dark energy, then there is no point in measuring the dark energy equation-of-state parameter $w=p / \rho$, where $p$ and $\rho$ are the pressure and energy density of the dark energy, respectively.

String theory seems to lead to many possible low-energy EFTs, so conversely one can ask what criteria a given low-energy EFT should satisfy in order to be contained in the string landscape. For the last decade, several criteria of this kind, dubbed swampland conjectures, have been proposed [9-11]. These can have important cosmological implications. For instance, one of the relatively wellestablished conjectures is the distance swampland conjecture [10,12-24], which implies that scalar fields in a low-energy EFT of a consistent theory of quantum gravity cannot have field excursions much larger than the Planck scale since otherwise an infinite tower of states becomes exponentially light and the validity of the EFT breaks down. In other words, one has the constraint [25]

$$
\Delta \phi \lesssim \alpha M_{\mathrm{Pl}}, \quad \alpha \approx O(1)
$$

In the context of inflation, field excursions are related to the tensor-to-scalar ratio $r$ by the Lyth bound [26], 


$$
\frac{\Delta \phi}{M_{\mathrm{Pl}}} \simeq \sqrt{\frac{r}{8}} \mathcal{N}
$$

where $\mathcal{N}$ is the number of $e$-folds of inflationary expansion. Clearly the distance conjecture, Eq. (1), limits the possibility of measuring tensor modes and hence primordial $B$-modes in the cosmic microwave background (CMB). Naively, with $\mathcal{N} \gtrsim 50$, we find $r \lesssim 0.003$, which is on the edge of observability for future experiments $[27,28]$.

The attempts to construct de Sitter solutions or inflationary models in string theory [7,29-39] have sparked discussions on various issues with such constructions, as well as no-go theorems [40-65]. Motivated by the obstructions encountered in various attempts, the de Sitter swampland conjecture was proposed [66], which states that the scalar potential of a low-energy limit of quantum gravity must satisfy

$$
M_{\mathrm{Pl}}|\nabla V| \geq c V, \quad c \approx O(1)>0
$$

where $\nabla$ denotes the gradient with respect to the field space, and the norm of the gradient is defined by the metric on field space. Whether the conjecture holds true is still an open debate [67-87]. Yet, even before the debate is settled, it is interesting and important to investigate both its consequences in cosmology and potential modifications or extensions [88-124]. The primary implication of this condition is that the observed positive energy density of our Universe should correspond to the potential of a rolling quintessence field rather than a positive $\Lambda$ [125]. The fact that one can easily embed any quintessence model into supergravity $[126,127]$ in a rather simple fashion, despite the fact that supersymmetry breaking generically spoils the flatness of the quintessence potential, is also encouraging. This raises the hope that $w \neq-1$ might be detected.

The de Sitter conjecture forbids (meta)stable vacua with positive energy density, so it is not surprising that the inflationary paradigm has apparent conflicts with the conjecture and one may call for a paradigm shift. Nonetheless, one can also adopt a conservative approach and regard the conjecture as a parametric constraint where the inequality holds but the number $c$ may not be strictly $\mathcal{O}(1)$ [99]. From this perspective, constraints on inflation can then be used to constrain $c$.

However, if we follow this route, the optimism that one can observe $w \neq-1$ is greatly diminished. To see this, recall that in single-field slow-roll inflation, the slow-roll parameters of the potential are defined as

$$
\epsilon_{V} \equiv \frac{M_{\mathrm{Pl}}^{2}}{2}\left(\frac{V^{\prime}}{V}\right)^{2}, \quad \eta_{V} \equiv M_{\mathrm{Pl}}^{2} \frac{V^{\prime \prime}}{V},
$$

where the primes denote derivatives with respect to the inflaton. The distance conjecture limits the inflaton field excursion $\Delta \phi \approx \sqrt{2 \epsilon_{V}} \mathcal{N} \lesssim \mathcal{O}(1)$, and therefore the necessary number of $e$-folds $\mathcal{N} \approx 50$ forces $c \lesssim \sqrt{2 \epsilon_{V}} \lesssim$ $\mathcal{N}^{-1} \sim 0.02$. On the other hand, the number $c$ in Eq. (3) is meant to be universal in a given EFT. Therefore, the current accelerating expansion must involve a quintessence field $Q$ whose potential $V_{Q}$ must satisfy

$1+w=\frac{2\left(V_{Q}^{\prime}\right)^{2}}{\left(V_{Q}^{\prime}\right)^{2}+6 V_{Q}^{2}}>\frac{2 c^{2}}{6+c^{2}} \equiv \Delta \gtrsim 1.33 \times 10^{-4}$.

Although this does not exclude observable quintessence, given the fact that so far almost all observations are consistent with a cosmological constant, such a small lower bound on a possible deviation of $w$ from -1 makes it questionable if it is worthwhile to push the sensitivity of the observations further. We may never know whether the Universe is de Sitter or quintessence.

However, the original de Sitter conjecture, Eq. (3), was so strong that even the Higgs potential was in tension with it [98]. The conjecture was also in tension with the wellunderstood supersymmetric AdS solutions [81]. Recently the refined de Sitter swampland conjecture was proposed $[101,128]$, which states that the scalar potential of a lowenergy theory that can be consistently coupled to quantum gravity should satisfy either

$$
\begin{gathered}
M_{\mathrm{Pl}}|\nabla V| \geq c V, \quad c \approx O(1)>0, \\
\text { or } \\
M_{\mathrm{Pl}}^{2} \min \left(\nabla_{i} \nabla_{j} V\right) \leq-c^{\prime} V, \quad c^{\prime} \approx O(1)>0,
\end{gathered}
$$

where $\min (\ldots)$ denotes the minimum eigenvalue of the Hessian $\nabla_{i} \nabla_{j} V$ in an orthonormal frame of the scalar field space. With this refinement, the aforementioned conflicts with the Higgs potential and the SUSY AdS solutions are resolved. The refined conjecture also raises new possibilities for inflation. In particular, one can evade the strict bound on $c$ arising from the distance conjecture by having the scalar potential satisfy the second condition, Eq. (7), of the new conjecture during part (or all) of inflation. As such, one may regain the hope that observable time-varying dark energy with $w \neq-1$ can be obtained. See also [129] for a recent discussion on $w$ considering the refined $\mathrm{dS}$ conjecture.

\section{SINGLE-FIELD SLOW-ROLL INFLATION MODELS}

Due to the above tension between the de Sitter conjecture and the requirements of inflation, we assume that the inflaton potential switches from one de Sitter condition to another as the inflaton rolls, an idea also utilized in [117]. To be specific, we take the following step-function approach to keep the discussion general and simple: we apply the first condition, Eq. (6), for the initial $\mathcal{N}_{1} e$-folds and apply the second condition, Eq. (7), for the remaining 
$\mathcal{N}_{2}=\mathcal{N}_{\text {tot }}-\mathcal{N}_{1} e$-folds. In our analysis we set $\mathcal{N}_{\text {tot }}=50$. We assume $\epsilon_{V}$ and $\eta_{V}$ are approximately constant for each interval so that we have

$$
\sqrt{2 \epsilon_{V}^{(1)}} \geq c \quad \text { and } \quad \eta_{V}^{(2)} \leq-c^{\prime}
$$

Additionally, Eq. (1) requires that

$$
\sqrt{2 \epsilon_{V}^{(1)}} \mathcal{N}_{1}+\sqrt{2 \epsilon_{V}^{(2)}} \mathcal{N}_{2} \leq \alpha \sim O(1)
$$

To maximize $c$, we assume $\epsilon_{V}^{(2)}<10^{-4}$ so that the contribution of the second era to Eq. (1) is negligible. Combining Eqs. (8) and (9), we have

$$
c<\frac{\alpha-\sqrt{2 \epsilon_{V}^{(2)}} \mathcal{N}_{2}}{\mathcal{N}_{1}} .
$$

We can also obtain a bound for $c^{\prime}$ from the spectral tilt $n_{s}=1-2 \epsilon-\eta$, where the Hubble slow-roll parameters are

$$
\epsilon=-\frac{\dot{H}}{H^{2}}, \quad \eta=\frac{\dot{\epsilon}}{H \epsilon} .
$$

For single-field inflation models, these are related to the slow-roll parameters of the potential as $\epsilon_{V}=\epsilon$ and $\eta_{V}=2 \epsilon-\frac{1}{2} \eta$. Therefore, we can constrain $\eta_{V}$ and hence the second parameter of the refined de Sitter conjecture as

$$
c^{\prime}<\frac{1}{2}\left(1-n_{s}(k)-6 \epsilon_{V}^{(2)}\right),
$$

where we allow for a $k$-dependent spectral tilt. Since we assume $\epsilon_{V}^{(2)}$ is small, our bounds simplify to

$$
\left(c^{\prime}, c\right)<\left(\frac{1-n_{s}(k)}{2}, \frac{\alpha}{\mathcal{N}_{1}}\right) .
$$

Equation (13) is valid until $\mathcal{N}_{1}=\mathcal{N}_{\text {tot }}$, at which point the derivation on the bound of $c^{\prime}$ above no longer applies, and the only constraint one finds is that $c<\alpha / \mathcal{N}_{\text {tot }}$. To proceed, we utilize the Planck analysis based on TT, TE, $\mathrm{EE}$, lowE, lensing and $\mathrm{BAO}[3]$, which gives

$$
\begin{gathered}
d n_{s} / d \ln k=-0.0041 \pm 0.0067, \\
n_{s}=0.9659 \pm 0.0040,
\end{gathered}
$$

at $k_{*}=0.05 \mathrm{Mpc}^{-1}$. We add errors in quadrature, ignoring correlations, and use

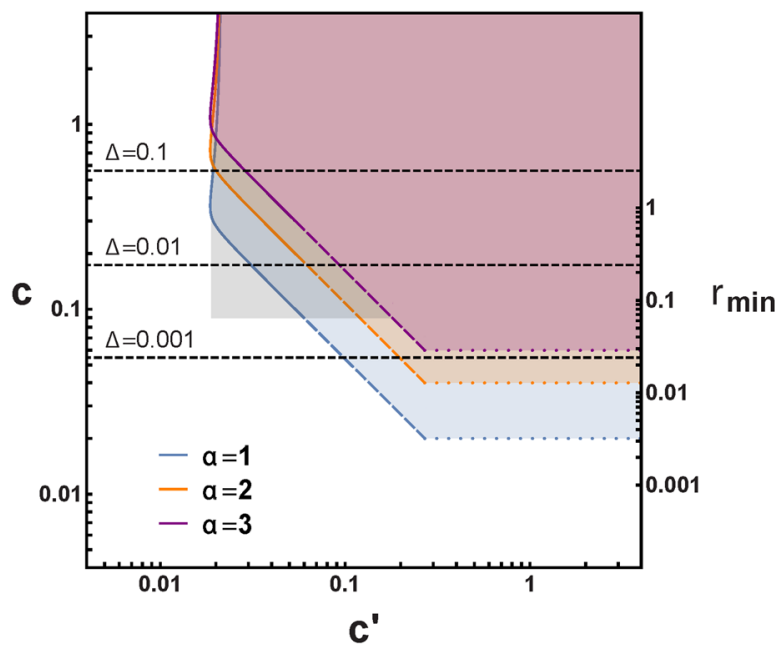

FIG. 1. Bounds on swampland parameters for generic singlefield inflation models at the $1 \sigma$ level assuming the running of $n_{s}$ can be extended to $\mathcal{N}_{\text {tot }}=50 e$-folds. The unshaded region is the allowed parameter space. The solid lines are for $\mathcal{N}_{1} \leq 10$, the dashed lines are for $10<\mathcal{N}_{1}<50$, and the horizontal dotted lines correspond to $\mathcal{N}_{1}=50$; i.e. the first constraint, Eq. (6), applies to the whole inflationary period. The values of $c$ excluded by [131] are shaded in grey. We use the distance conjecture with $\Delta \phi \leq \alpha M_{\mathrm{Pl}}$ and display the minimum values for $1+w \geq \Delta$ with black dashed lines. With the original de Sitter conjecture, $c$ has to be below the dotted horizontal lines, but there are no constraints on $c^{\prime}$.

$$
\begin{aligned}
n_{s}(k)= & 0.9659-0.0041 \ln \frac{k}{k_{*}} \\
& \pm \sqrt{(0.0040)^{2}+\left(0.0067 \ln \frac{k}{k_{*}}\right)^{2}} .
\end{aligned}
$$

A smaller $n_{s}$ allows for larger $c^{\prime}$ in Eq. (13), so we take the $1 \sigma$ allowed lower end in order to place our bounds. The weak correlation between $n_{s}$ and $d n_{s} / d \ln k$ we see in Fig. 26 of [3] actually works in our favor, and ignoring the correlation is therefore the more conservative approach (i.e., it gives a smaller allowed range) [130]. Using the simple relationship $\mathcal{N}_{1}=\ln \left(k / a_{0} H_{0}\right)$, where $a_{0}$ is the present scale factor and $H_{0}$ is the present Hubble scale, we can constrain the swampland parameters in single-field inflation as shown in Fig. 1. The current CMB constraints on the spectral index and its running are limited to $\mathcal{N}_{1} \lesssim 10$. This range is denoted by the solid lines in Fig. 1. Beyond this there are no strong observational constraints, and we extend our analysis by extrapolating Eq. (16) to $\mathcal{N}_{1} \geq 10$ shown by the dashed lines in Fig. 1. The unshaded regions indicate values of $\left(c^{\prime}, c\right)$ that satisfy the above inequalities. The vertical asymptotes correspond to satisfying Eq. (7) for the entirety of the inflationary epoch, $\mathcal{N}_{1}=0$, so that $c$ is left completely arbitrary but $c^{\prime}$ has a strict upper bound that is much less than the $\mathcal{O}(1)$ expectation. The horizontal dotted lines correspond to 
satisfying the first constraint, Eq. (6), for all of inflation, $\mathcal{N}_{2}=0$, which leaves $c^{\prime}$ arbitrary but severely limits $c$. The horizontal black dashed lines indicate the lowest values of $c$ that yield the given $\Delta$ defined in Eq. (5) as the lower bound on $1+w$ from the constraint, Eq. (6). Finally, the grey region excludes values of $c$ that may satisfy Eq. (13), depending on the value of $\alpha$, but conflicts with the constraint $r_{0.002}<0.064$ [131], as $r=16 \epsilon \geq 8 c^{2}$. The grey excluded region has a left vertical boundary since the constraint applies only to $k>0.002 \mathrm{Mpc}^{-1}$.

We also comment on the observability of the tensor mode $r$. The swampland distance conjecture, Eq. (1), combined with the Lyth bound, Eq. (2), is normally believed to disfavor observably large $r$, assuming $\alpha \approx 1$. The best sensitivity anticipated in the future is $r \sim 10^{-3}$ $[27,28]$. There is a parameter region in Fig. 1 where $r \geq$ $r_{\min } \equiv 8 c^{2}$ is close to the current observational bound. Physically this is because, in our spirit of a step function approximation, we can allow for a brief initial period, say $\mathcal{N}_{0} \sim 4$, where the upper bound on $\epsilon$ from the distance conjecture, $\epsilon \lesssim \mathcal{N}_{0}^{-2} / 2 \sim 0.03$, is relaxed. Thus, it is possible to have $r$ large enough to saturate the observational bound at low $\ell$. This is encouraging, especially for spaceborne CMB $B$-mode experiments such as LiteBIRD [28].

\section{MULTIFIELD SLOW-ROLL INFLATION MODELS}

The constraints discussed above are due to the tight relations between $n_{s}, \epsilon_{V}, \eta_{V}$, and $r$ in single-field slow-roll inflation models. It is natural to ask whether the constraints can be relaxed in multifield models. In our analysis below, we take the conservative assumption that the swampland distance conjecture applies to the proper length of the trajectory, instead of the geodesic distance between the starting and ending points in the field space.

We discuss here a class of multifield models where directions orthogonal to the slow-roll direction are massive, $M \gtrsim H$. The inflaton therefore rolls near the bottom of the valley, which has "bends" in the multidimensional field space. The main difference here is that the local angular velocities of the inflaton around the bends can modify the effective sound speed $c_{s}$ of fluctuations. As a result, we have the modified relation [132]

$$
\begin{aligned}
12 \eta_{V}= & \left(c_{s}^{-2}-1\right) \frac{M^{2}}{H^{2}}+2 \frac{M^{2}}{H^{2}}+3(4 \epsilon-\eta) \\
& -2 \sqrt{\left(\frac{M^{2}}{H^{2}}-\frac{3}{2}(4 \epsilon-\eta)\right)^{2}+9\left(c_{s}^{-2}-1\right) \frac{M^{2}}{H^{2}}} .
\end{aligned}
$$

Here, $\eta_{V}$ is the minimum eigenvalue of the Hessian, $M$ is the effective mass of the field orthogonal to the slow-roll direction, and $c_{s}$ is given by

$$
c_{s}^{-2}=1+\frac{4 \Omega^{2}}{M^{2}}
$$

where $\Omega$ is the local angular velocity describing the bend of the inflaton trajectory in the potential. Note that in the limit $\Omega \rightarrow 0$, the sound speed reduces to unity and $\eta_{V}$ to the expression of the single-field models. Allowing for a significant deviation of $c_{s}$ from unity relaxes the constraints on $\left(c, c^{\prime}\right)$, as shown in Fig. 2, where we set $M=H$. This allows for larger values of $c$ and $c^{\prime}$ compared to the singlefield case, which are preferred by the swampland conjecture. Note that lowering the sound speed further will not achieve $\mathcal{O}(1)$ values for $c^{\prime}$ because our scenario relies on having negative $\eta_{V}$. As $c_{s}$ is reduced from unity, $\eta_{V}$ initially becomes more negative and widens the allowed parameter space. Beyond some critical value $c_{s} \approx 0.3$, further reduction of $c_{s}$ makes $\eta_{V}$ less negative, thereby narrowing the allowed parameter space. For $c_{s} \lesssim 0.2, \eta_{V}$ becomes positive and our analysis no longer holds. Empirically, we find that $c_{s} \sim 0.24$ maximizes the allowed parameter region in the $\left(c^{\prime}, c\right)$ plane. The grey shaded regions again correspond to experimental constraints on $r=16 \epsilon c_{s}$, but their area is greatly reduced as $c_{s}$ decreases.

It is also interesting to note that we expect primordial equilateral and orthogonal non-Gaussianities once $c_{s} \neq 1$ in this class of models [132],

$$
f_{\mathrm{NL}}^{\mathrm{equil}}=-\left(c_{s}^{-2}-1\right)\left(0.275+0.078 c_{s}^{2}\right),
$$

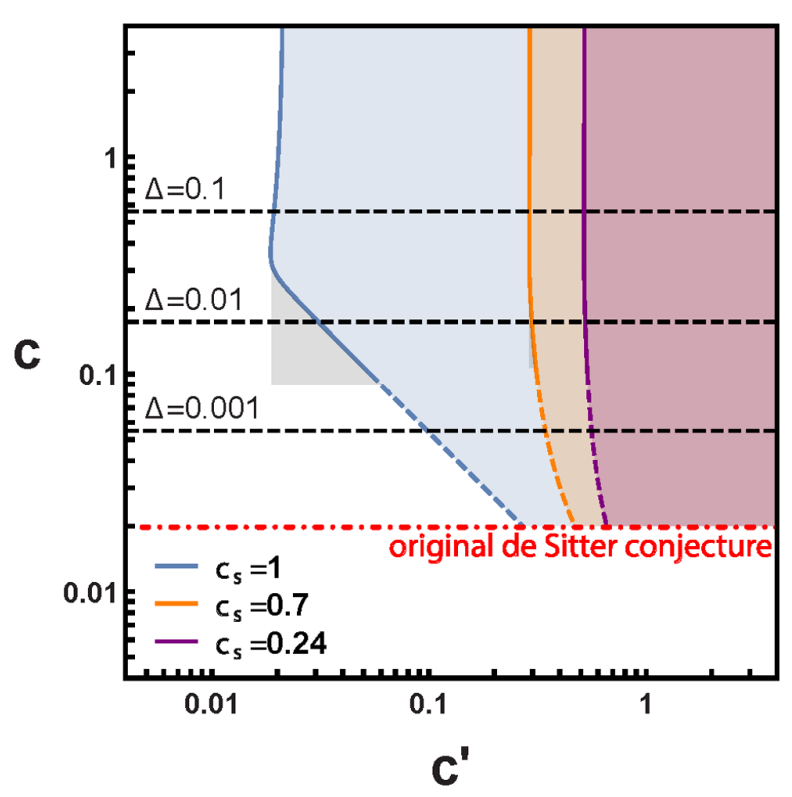

FIG. 2. Bounds on swampland parameters for generic multifield inflation models. We take $\alpha=1$ and $M=H$. Here, $c_{s}$ is the sound speed for fluctuations, and the rest is the same as in Fig. 1. With the original de Sitter conjecture, Eq. (3), and single-field slow-roll models, $c$ has to be below the red dot-dashed horizontal line. 


$$
f_{\mathrm{NL}}^{\text {ortho }}=\left(c_{s}^{-2}-1\right)\left(0.0159-0.0167 c_{s}^{2}\right) .
$$

Here we have ignored the third order parameter. The current observational constraint on the sound speed is $c_{s} \geq 0.024$ [see Eq. (89) of [133] ], which is an order of magnitude below the limit we can reach in our setup, as shown in Fig. 2. Future observations combining CMB lensing, galaxy and $21 \mathrm{~cm}$ surveys, Lyman $\alpha$ forest, etc., have the potential to improve the constraint on $f_{\mathrm{NL}}$ by an order of magnitude or more [134].

\section{IMPLICATIONS FOR DARK ENERGY}

The de Sitter conjecture states that constants $c$ and $c^{\prime}$ are universal and should apply to all sectors in a given EFT. Therefore, we can use inflationary physics to get a handle on the values of $c$ and $c^{\prime}$ and apply this knowledge to the quintessence potential $V_{Q}$. When this argument is applied to single-field inflation models with conjectures Eqs. (3) and (1), one deduces that there may be little hope in finding $w \neq-1$ due to the small lower bound seen in Eq. (5). This depressing outlook is drastically changed in light of Eqs. (6) and (7), as Fig. 1 illustrates. We see that the refined de Sitter conjecture has allowed for the possibility of having $\Delta$ bounded from below such that it must be larger than a few percent and should be observable to experiments. Current and future experiments, such as DES [135], HSC [136], DESI [137], PFS [138], LSST [139], Euclid [140], and WFIRST [141], are aiming for an accuracy of about a percent in $w$. The cost for this is that $c^{\prime}$ must be much lower than the $\mathcal{O}(1)$ expectation of $[101,128]$ in the single-field case. This seems to indicate that single-field inflation falls more in line with the modified de Sitter conjecture discussed in [92], where the smallest Hessian eigenvalue needs to only be negative when $|\nabla V|<c V$.

This state of affairs is altered by considering multifield inflation models. Not only could $\Delta$ be forced to be as large as several percent, it is also possible to have both $c$ and $c^{\prime}$ approximately $\mathcal{O}(1)$ as long as the sound speed is low enough, as seen in Fig. 2. In either the single-field or multifield scenario, a better theoretical understanding of the magnitude of $c^{\prime}$ is essential to understand the consistency of the swampland conjectures and inflation.

\section{CONCLUSIONS}

In this paper, we studied the consequences of the latest swampland conjecture on inflation and dark energy. The original de Sitter conjecture raised the hope that measuring the dark energy equation of state $w$ would be promising but simultaneously dashed that hope since the consistency with single-field inflation suggests that the deviation from $w=-1$ would likely be unobservable. As we have shown, this situation is much more encouraging with the refined de Sitter conjecture. Not only could $w \neq-1$ be observable even with a single-field inflationary scenario, but tensor modes could be as well. If one considers multifield inflationary scenarios, then the prospect for observing $w \neq-1$ is better, and one gains improved agreement with the swampland conjectures.

\section{ACKNOWLEDGMENTS}

We would like to thank Katelin Schutz for useful discussions and comments on the manuscript. H. M., C. -I. C., and J. M. L. were supported by the U.S. DOE Contract No. DE-AC02-05CH11231. H. M. was also supported by the NSF Grant No. PHY-1638509, by the JSPS Grant-in-Aid for Scientific Research (C) (17K05409), MEXT Grant-in-Aid for Scientific Research on Innovative Areas (15H05887 and 15K21733), by World Premier International Research Center Initiative, MEXT, Japan, and Hamamatsu Photonics as Hamamatsu Professor.
[1] S. Perlmutter et al. (Supernova Cosmology Project Collaboration), Measurements of $\Omega$ and $\Lambda$ from 42 high redshift supernovae, Astrophys. J. 517, 565 (1999).

[2] A. G. Riess et al. (Supernova Search Team Collaboration), Observational evidence from supernovae for an accelerating universe and a cosmological constant, Astron. J. 116, 1009 (1998).

[3] N. Aghanim et al. (Planck Collaboration), Planck 2018 results. VI. Cosmological parameters, arXiv:1807.06209.

[4] S. Weinberg, The cosmological constant problem, Rev. Mod. Phys. 61, 1 (1989).

[5] R. Bousso and J. Polchinski, Quantization of four form fluxes and dynamical neutralization of the cosmological constant, J. High Energy Phys. 06 (2000) 006.
[6] S. B. Giddings, S. Kachru, and J. Polchinski, Hierarchies from fluxes in string compactifications, Phys. Rev. D 66 , 106006 (2002).

[7] S. Kachru, R. Kallosh, A. D. Linde, and S. P. Trivedi, de Sitter vacua in string theory, Phys. Rev. D 68, 046005 (2003).

[8] S. Weinberg, Anthropic Bound on the Cosmological Constant, Phys. Rev. Lett. 59, 2607 (1987).

[9] C. Vafa, The string landscape and the swampland, arXiv: hep-th/0509212.

[10] H. Ooguri and C. Vafa, On the geometry of the string landscape and the swampland, Nucl. Phys. B766, 21 (2007).

[11] N. Arkani-Hamed, L. Motl, A. Nicolis, and C. Vafa, The string landscape, black holes and gravity as the weakest force, J. High Energy Phys. 06 (2007) 060. 
[12] E. Palti, On natural inflation and moduli stabilisation in string theory, J. High Energy Phys. 10 (2015) 188.

[13] F. Baume and E. Palti, Backreacted axion field ranges in string theory, J. High Energy Phys. 08 (2016) 043.

[14] D. Klaewer and E. Palti, Super-Planckian spatial field variations and quantum gravity, J. High Energy Phys. 01 (2017) 088.

[15] I. Valenzuela, Backreaction issues in axion monodromy and Minkowski 4-forms, J. High Energy Phys. 06 (2017) 098.

[16] R. Blumenhagen, I. Valenzuela, and F. Wolf, The swampland conjecture and F-term axion monodromy inflation, J. High Energy Phys. 07 (2017) 145.

[17] E. Palti, The weak gravity conjecture and scalar fields, J. High Energy Phys. 08 (2017) 034.

[18] D. Lüst and E. Palti, Scalar fields, hierarchical UV/IR mixing and the weak gravity conjecture, J. High Energy Phys. 02 (2018) 040.

[19] A. Hebecker, P. Henkenjohann, and L. T. Witkowski, Flat monodromies and a moduli space size conjecture, J. High Energy Phys. 12 (2017) 033.

[20] M. Cicoli, D. Ciupke, C. Mayrhofer, and P. Shukla, A geometrical upper bound on the inflaton range, J. High Energy Phys. 05 (2018) 001.

[21] T. W. Grimm, E. Palti, and I. Valenzuela, Infinite distances in field space and massless towers of states, J. High Energy Phys. 08 (2018) 143.

[22] B. Heidenreich, M. Reece, and T. Rudelius, Emergence of Weak Coupling at Large Distance in Quantum Gravity, Phys. Rev. Lett. 121, 051601 (2018).

[23] R. Blumenhagen, D. Klaewer, L. Schlechter, and F. Wolf, The refined swampland distance conjecture in Calabi-Yau moduli spaces, J. High Energy Phys. 06 (2018) 052.

[24] S.-J. Lee, W. Lerche, and T. Weigand, Tensionless strings and the weak gravity conjecture, J. High Energy Phys. 10 (2018) 164.

[25] We note that $\alpha$ can be greater than unity. We restrict ourselves to a more conservative approach and keep $\alpha \sim 1$, but values as large as $2 \log \left(M_{\mathrm{pl}} / H_{\text {inf }}\right)$ could be permitted.

[26] E. J. Copeland, A. R. Liddle, D. H. Lyth, E. D. Stewart, and D. Wands, False vacuum inflation with Einstein gravity, Phys. Rev. D 49, 6410 (1994).

[27] K. N. Abazajian et al. (CMB-S4 Collaboration), 1st ed., CMB-S4 Science Book, https://inspirehep.net/record/ 1490867.

[28] A. Suzuki et al., The LiteBIRD satellite mission-subKelvin instrument, J. Low Temp. Phys. 193, 1048 (2018).

[29] S. Kachru, R. Kallosh, A. D. Linde, J. M. Maldacena, L. P. McAllister, and S. P. Trivedi, Towards inflation in string theory, J. Cosmol. Astropart. Phys. 10 (2003) 013.

[30] E. Silverstein and D. Tong, Scalar speed limits and cosmology: Acceleration from D-cceleration, Phys. Rev. D 70, 103505 (2004).

[31] V. Balasubramanian, P. Berglund, J. P. Conlon, and F. Quevedo, Systematics of moduli stabilisation in CalabiYau flux compactifications, J. High Energy Phys. 03 (2005) 007.

[32] D. Baumann, A. Dymarsky, I. R. Klebanov, J. M. Maldacena, L. P. McAllister, and A. Murugan, On D3-brane potentials in compactifications with fluxes and wrapped D-branes, J. High Energy Phys. 11 (2006) 031.

[33] A. Westphal, de Sitter string vacua from Kahler uplifting, J. High Energy Phys. 03 (2007) 102.

[34] D. Baumann, A. Dymarsky, I. R. Klebanov, and L. McAllister, Towards an explicit model of D-brane inflation, J. Cosmol. Astropart. Phys. 01 (2008) 024.

[35] X. Dong, B. Horn, E. Silverstein, and G. Torroba, Micromanaging de Sitter holography, Classical Quantum Gravity 27, 245020 (2010).

[36] M. Rummel and A. Westphal, A sufficient condition for de Sitter vacua in type IIB string theory, J. High Energy Phys. 01 (2012) 020.

[37] J. Blåbäck, U. Danielsson, and G. Dibitetto, Accelerated universes from type IIA compactifications, J. Cosmol. Astropart. Phys. 03 (2014) 003.

[38] M. Cicoli, D. Klevers, S. Krippendorf, C. Mayrhofer, F. Quevedo, and R. Valandro, Explicit de Sitter flux vacua for global string models with chiral matter, J. High Energy Phys. 05 (2014) 001.

[39] M. Cicoli, F. Quevedo, and R. Valandro, de Sitter from T-branes, J. High Energy Phys. 03 (2016) 141.

[40] J. M. Maldacena and C. Nunez, Supergravity description of field theories on curved manifolds and a no go theorem, Int. J. Mod. Phys. A 16, 822 (2001).

[41] P. K. Townsend, Cosmic acceleration and M theory, in Mathematical Physics. Proceedings, 14th International Congress, ICMP 2003, Lisbon, Portugal, 2003 (World Scientific, Hackensack, USA, 2003), pp. 655-662, http:// inspirehep.net/record/728128.

[42] M. P. Hertzberg, S. Kachru, W. Taylor, and M. Tegmark, Inflationary constraints on type IIA string theory, J. High Energy Phys. 12 (2007) 095.

[43] L. Covi, M. Gomez-Reino, C. Gross, J. Louis, G. A. Palma, and C. A. Scrucca, de Sitter vacua in no-scale supergravities and Calabi-Yau string models, J. High Energy Phys. 06 (2008) 057.

[44] P. McGuirk, G. Shiu, and Y. Sumitomo, Nonsupersymmetric infrared perturbations to the warped deformed conifold, Nucl. Phys. B842, 383 (2011).

[45] C. Caviezel, P. Koerber, S. Kors, D. Lüst, T. Wrase, and M. Zagermann, On the cosmology of type IIA compactifications on SU(3)-structure manifolds, J. High Energy Phys. 04 (2009) 010.

[46] C. Caviezel, T. Wrase, and M. Zagermann, Moduli stabilization and cosmology of type IIB on SU(2)-structure orientifolds, J. High Energy Phys. 04 (2010) 011.

[47] B. de Carlos, A. Guarino, and J. M. Moreno, Flux moduli stabilisation, supergravity algebras and no-go theorems, J. High Energy Phys. 01 (2010) 012.

[48] T. Wrase and M. Zagermann, On classical de Sitter vacua in string theory, Fortsch. Phys. 58, 906 (2010).

[49] G. Shiu and Y. Sumitomo, Stability constraints on classical de Sitter vacua, J. High Energy Phys. 09 (2011) 052.

[50] S. R. Green, E. J. Martinec, C. Quigley, and S. Sethi, Constraints on string cosmology, Classical Quantum Gravity 29, 075006 (2012).

[51] F. F. Gautason, D. Junghans, and M. Zagermann, On cosmological constants from alpha'-corrections, J. High Energy Phys. 06 (2012) 029. 
[52] I. Bena, M. Grana, S. Kuperstein, and S. Massai, PolchinskiStrassler does not uplift Klebanov-Strassler, J. High Energy Phys. 09 (2013) 142.

[53] J. Blåbäck, U. H. Danielsson, and T. Van Riet, Resolving anti-brane singularities through time-dependence, J. High Energy Phys. 02 (2013) 061.

[54] I. Bena, M. Grana, S. Kuperstein, and S. Massai, Giant tachyons in the landscape, J. High Energy Phys. 02 (2015) 146.

[55] U. H. Danielsson and T. Van Riet, Fatal attraction: More on decaying anti-branes, J. High Energy Phys. 03 (2015) 087.

[56] D. Kutasov, T. Maxfield, I. Melnikov, and S. Sethi, Constraining de Sitter space in string theory, Phys. Rev. Lett. 115, 071305 (2015).

[57] C. Quigley, Gaugino condensation and the cosmological constant, J. High Energy Phys. 06 (2015) 104.

[58] K. Dasgupta, R. Gwyn, E. McDonough, M. Mia, and R. Tatar, de Sitter vacua in type IIB string theory: Classical solutions and quantum corrections, J. High Energy Phys. 07 (2014) 054.

[59] D. Junghans and M. Zagermann, A universal tachyon in nearly no-scale de Sitter compactifications, J. High Energy Phys. 07 (2018) 078.

[60] D. Junghans, Tachyons in classical de Sitter vacua, J. High Energy Phys. 06 (2016) 132.

[61] D. Andriot and J. Blåbäck, Refining the boundaries of the classical de Sitter landscape, J. High Energy Phys. 03 (2017) 102; Erratum 03 (2018) 83.

[62] J. Moritz, A. Retolaza, and A. Westphal, Toward de Sitter space from ten dimensions, Phys. Rev. D 97, 046010 (2018).

[63] S. Sethi, Supersymmetry breaking by fluxes, J. High Energy Phys. 10 (2018) 022.

[64] D. Andriot, On classical de Sitter and Minkowski solutions with intersecting branes, J. High Energy Phys. 03 (2018) 054.

[65] U. H. Danielsson and T. Van Riet, What if string theory has no de Sitter vacua?, Int. J. Mod. Phys. D 27, 1830007 (2018).

[66] G. Obied, H. Ooguri, L. Spodyneiko, and C. Vafa, de Sitter space and the swampland, arXiv:1806.08362.

[67] R. Kallosh and T. Wrase, Emergence of spontaneously broken supersymmetry on an anti-D3-brane in KKLT dS vacua, J. High Energy Phys. 12 (2014) 117.

[68] S. Ferrara, R. Kallosh, and A. Linde, Cosmology with nilpotent superfields, J. High Energy Phys. 10 (2014) 143.

[69] E. A. Bergshoeff, K. Dasgupta, R. Kallosh, A. Van Proeyen, and T. Wrase, D-3 and dS, J. High Energy Phys. 05 (2015) 058.

[70] E. A. Bergshoeff, D. Z. Freedman, R. Kallosh, and A. Van Proeyen, Pure de Sitter supergravity, Phys. Rev. D 92, 085040 (2015); Erratum 93, 069901 (2016).

[71] F. Hasegawa and Y. Yamada, Component action of nilpotent multiplet coupled to matter in 4 dimensional $\mathcal{N}=1$ supergravity, J. High Energy Phys. 10 (2015) 106.

[72] R. Kallosh, F. Quevedo, and A. M. Uranga, String theory realizations of the nilpotent Goldstino, J. High Energy Phys. 12 (2015) 039.

[73] J. Polchinski, Brane/antibrane dynamics and KKLT stability, arXiv:1509.05710.

[74] R. Kallosh, B. Vercnocke, and T. Wrase, String theory origin of constrained multiplets, J. High Energy Phys. 09 (2016) 063.
[75] L. Aalsma, M. Tournoy, J. P. Van Der Schaar, and B. Vercnocke, A supersymmetric embedding of anti-brane polarization, arXiv:1807.03303.

[76] M. Cicoli, S. de Alwis, A. Maharana, F. Muia, and F. Quevedo, de Sitter vs quintessence in string theory, Fortsch. Phys. 67, 1800079 (2019).

[77] K. Dasgupta, M. Emelin, E. McDonough, and R. Tatar, Quantum corrections and the de Sitter swampland conjecture, J. High Energy Phys. 01 (2019) 145.

[78] C. Roupec and T. Wrase, de Sitter extrema and the swampland, Fortsch. Phys. 67, 1800082 (2019).

[79] D. Andriot, New constraints on classical de Sitter: Flirting with the swampland, Fortsch. Phys. 67, 1800103 (2019).

[80] D. Andriot, On the de Sitter swampland criterion, Phys. Lett. B 785, 570 (2018).

[81] J.P. Conlon, The de Sitter swampland conjecture and supersymmetric AdS vacua, Int. J. Mod. Phys. A 33, 1850178 (2018).

[82] Y. Akrami, R. Kallosh, A. Linde, and V. Vardanyan, The landscape, the swampland and the era of precision cosmology, Fortsch. Phys. 67, 1800075 (2019).

[83] R. Kallosh, A. Linde, E. McDonough, and M. Scalisi, de Sitter vacua with a nilpotent superfield, Fortsch. Phys. 67, 1800068 (2019).

[84] S. Kachru and S. Trivedi, A comment on effective field theories of flux vacua, Fortsch. Phys. 67, 1800086 (2019).

[85] J. Moritz, A. Retolaza, and A. Westphal, On uplifts by warped anti-D3-branes, Fortsch. Phys. 67, 1800098 (2019).

[86] I. Bena, E. Dudas, M. Graña, and S. Lüst, Uplifting runaways, Fortsch. Phys. 67, 1800100 (2019).

[87] F. F. Gautason, V. Van Hemelryck, and T. Van Riet, The tension between 10D supergravity and dS uplifts, Fortsch. Phys. 67, 1800091 (2019).

[88] K. Choi, D. Chway, and C. S. Shin, The dS swampland conjecture with the electroweak symmetry and QCD chiral symmetry breaking, J. High Energy Phys. 11 (2018) 142.

[89] L. Heisenberg, M. Bartelmann, R. Brandenberger, and A. Refregier, Dark energy in the swampland II, Fortsch. Phys. 67, 1800075 (2019).

[90] L. Heisenberg, M. Bartelmann, R. Brandenberger, and A. Refregier, Dark energy in the swampland, Phys. Rev. D 98, 123502 (2018).

[91] M.C.D. Marsh, The swampland, quintessence and the vacuum energy, Phys. Lett. B 789, 639 (2019).

[92] H. Murayama, M. Yamazaki, and T. T. Yanagida, Do we live in the swampland?, J. High Energy Phys. 12 (2018) 032.

[93] W. H. Kinney, S. Vagnozzi, and L. Visinelli, The zoo plot meets the swampland: Mutual (in)consistency of singlefield inflation, string conjectures, and cosmological data, Classical Quantum Gravity 36, 117001 (2019).

[94] O. Loaiza-Brito and O. Loaiza-Brito, Two-field axion inflation and the swampland constraint in the flux-scaling scenario, Fortsch. Phys. 67, 1800072 (2019).

[95] I. Ben-Dayan, Draining the swampland, Phys. Rev. D 99, 101301 (2019).

[96] H. Matsui and F. Takahashi, Eternal inflation and swampland conjectures, Phys. Rev. D 99, 023533 (2019). 
[97] O. E. Colgáin, M. H. P. M. Van Putten, and H. Yavartanoo, $H_{0}$ tension and the de Sitter swampland, Phys. Lett. B 793, 126 (2019).

[98] F. Denef, A. Hebecker, and T. Wrase, The dS swampland conjecture and the Higgs potential, Phys. Rev. D 98, 086004 (2018).

[99] M. Dias, J. Frazer, A. Retolaza, and A. Westphal, Primordial gravitational waves and the swampland, Fortsch. Phys. 67, 1800063 (2019).

[100] A. Kehagias and A. Riotto, A note on inflation and the swampland, Fortsch. Phys. 66, 1800052 (2018).

[101] S. K. Garg and C. Krishnan, Bounds on slow roll and the de Sitter swampland, arXiv:1807.05193.

[102] A. Achúcarro and G. A. Palma, The string swampland constraints require multi-field inflation, J. Cosmol. Astropart. Phys. 02 (2019) 041.

[103] S. Das, A note on single-field inflation and the swampland criteria, Phys. Rev. D 99, 083510 (2019).

[104] D. Wang, The multi-feature universe: Large parameter space cosmology and the swampland, arXiv:1809.04854.

[105] R. H. Brandenberger, Beyond standard inflationary cosmology, in Beyond Spacetime, edited by N. Huggett, K. Matsubara, and C. Wuethrich (Cambridge University Press, Cambridge, England, 2018).

[106] C. Han, S. Pi, and M. Sasaki, Quintessence saves Higgs instability, Phys. Lett. B 791, 314 (2019).

[107] R. Brandenberger, R. R. Cuzinatto, J. Fröhlich, and R. Namba, New scalar field quartessence, J. Cosmol. Astropart. Phys. 02 (2019) 043.

[108] K. Dimopoulos, Steep eternal inflation and the swampland, Phys. Rev. D 98, 123516 (2018).

[109] J. Ellis, B. Nagaraj, D. V. Nanopoulos, and K. A. Olive, de Sitter vacua in no-scale supergravity, J. High Energy Phys. 11 (2018) 110.

[110] C.-M. Lin, K.-W. Ng, and K. Cheung, Chaotic inflation on the brane and the swampland criteria, arXiv:1810.01644.

[111] K. Hamaguchi, M. Ibe, and T. Moroi, The swampland conjecture and the Higgs expectation value, J. High Energy Phys. 12 (2018) 023.

[112] M. Kawasaki and V. Takhistov, Primordial black holes and the string swampland, Phys. Rev. D 98, 123514 (2018).

[113] M. Motaharfar, V. Kamali, and R. O. Ramos, Warm way out of the swampland, Phys. Rev. D 99, 063513 (2019).

[114] A. Ashoorioon, Rescuing single field inflation from the swampland, Phys. Lett. B 790, 568 (2019).

[115] S. Das, Warm inflation in the light of swampland criteria, Phys. Rev. D 99, 063514 (2019).

[116] S.-J. Wang, Quintessential Starobinsky inflation and swampland criteria, Phys. Rev. D 99, 023529 (2019).

[117] H. Fukuda, R. Saito, S. Shirai, and M. Yamazaki, Phenomenological consequences of the refined swampland conjecture, Phys. Rev. D 99, 083520 (2019).

[118] A. Hebecker and T. Wrase, The asymptotic dS swampland conjecture-A simplified derivation and a potential loophole, Fortsch. Phys. 67, 1800097 (2019).

[119] Y. Olguin-Tejo, S. L. Parameswaran, G. Tasinato, and I. Zavala, Runaway quintessence, out of the swampland, J. Cosmol. Astropart. Phys. 01 (2019) 031.
[120] S. K. Garg, C. Krishnan, and M. Z. Zaz, Bounds on slow roll at the boundary of the landscape, J. High Energy Phys. 03 (2019) 029.

[121] S. C. Park, Minimal gauge inflation and the refined swampland conjecture, J. Cosmol. Astropart. Phys. 01 (2019) 053.

[122] J. Blåbäck, U. Danielsson, and G. Dibitetto, A new light on the darkest corner of the landscape, arXiv:1810.11365.

[123] R. Schimmrigk, The swampland spectrum conjecture in inflation, arXiv:1810.11699.

[124] C.-M. Lin, Type I hilltop inflation and the refined swampland criteria, Phys. Rev. D 99, 023519 (2019).

[125] P. Agrawal, G. Obied, P. J. Steinhardt, and C. Vafa, On the cosmological implications of the string swampland, Phys. Lett. B 784, 271 (2018).

[126] P. Brax, C. van de Bruck, J. Martin, and A.-C. Davis, Decoupling dark energy from matter, J. Cosmol. Astropart. Phys. 09 (2009) 032.

[127] C.-I. Chiang and H. Murayama, Building supergravity quintessence model, arXiv:1808.02279.

[128] H. Ooguri, E. Palti, G. Shiu, and C. Vafa, Distance and de Sitter conjectures on the swampland, Phys. Lett. B 788, 180 (2019).

[129] P. Agrawal and G. Obied, Dark energy and the refined de Sitter conjecture, arXiv:1811.00554.

[130] The Planck 2018 paper [3] also shows the analysis where they allow for the running of running $d^{2} n_{s} / d \ln k^{2}$. Unfortunately they do not show the correlation, and we cannot use it for our purposes. In fact, the extrapolation of $n_{s}(k)$ to small scales from the Planck data is most likely too restrictive, as the allowed range for the primordial power $P_{\zeta}(k)$ blows up for $k \gtrsim 0.2 \mathrm{Mpc}^{-1}$ (see Fig. 20 in [131]).

[131] Y. Akrami et al. (Planck Collaboration), Planck 2018 results. X. Constraints on inflation, arXiv:1807.06211.

[132] A. Hetz and G. A. Palma, Sound Speed of Primordial Fluctuations in Supergravity Inflation, Phys. Rev. Lett. 117, 101301 (2016).

[133] P. A. R. Ade et al. (Planck Collaboration), Planck 2015 results. XVII. Constraints on primordial non-Gaussianity, Astron. Astrophys. 594, A17 (2016).

[134] C. Lawrence et al. (Cosmic Microwave Background Stage 4 Concept Definition Task Force Collaboration), Report to the astronomy and astrophysics advisory committee, https://www.nsf.gov/mps/ast/aaac/cmb_s4/report/ CMBS4_final_report_NL.pdf.

[135] Dark Energy Survey Collaboration, https://www .darkenergysurvey.org.

[136] Hyper Suprime-Cam Collaboration, https://hsc.mtk.nao.ac .jp/ssp.

[137] Dark Energy Spectroscopic Instrument Collaboration, https://www.desi.lbl.gov.

[138] Prime Focus Spectrograph Collaboration, https://pfs.ipmu.jp.

[139] Large Synoptic Survey Telescope Collaboration, https:// www.lsstcorporation.org.

[140] Euclid Collaboration, https://www.euclid-ec.org.

[141] Wide Field Infrared Survey Telescope Collaboration, https://wfirst.gsfc.nasa.gov.

[142] See Supplemental Material at http://link.aps.org/ supplemental/10.1103/PhysRevD.100.043505 for depiction of the piecewise single-field inflation potential considered in the main text. 